\title{
AN APPROACH TO IMPROVEMENT THE USABILITY IN SOFTWARE PRODUCTS
}

\author{
Tawfig M. Abdelaziz, Abdelsalam M. Maatuk and Fatma Rajab \\ University of Benghazi, Faculty of Information Technology, Department of Software \\ Engineering
}

\begin{abstract}
One of the significantaspects of software quality is usability. It is one of the characteristics that judge by the success or failure of software applications. The most important risk facing the software applications is usability which may lead to the existence of a gap between users and systems. This may lead to system failure because of Poor design. This is due to the design is not based on the desires and requirements of the customer. To overcome these problems, this paper proposed an approach to improve usability of software applications to meet the needs of the customer and interacts with the user easily with an efficient and effective manner.The proposed approach is based prototyping technique due to itssimplicity and it does not require additional costs to elicit precise and complete requirement and design.
\end{abstract}

\section{KEYWORDS:}

Software quality, usability, user experience, design.

\section{INTRODUCTION}

Understand software quality is central to answer the significant asked question: what is quality? As soon as the concept of quality is understood the different structures of quality available on the market are easily understood. As follows, and before we start into the quality issues, we will spend some time to shed light onthe question: what is quality?As many well-known researchers have provided an answer to that question, we can classify that there are two key terms when discussing the meaning and definition of (software) quality [6].

In "Jurans's Quality Control Handbook" Joseph M. Juran provides two meanings to quality: The word quality has multiple meanings. Two of those meanings have influence over the use of the word:

Quality consists of the product characteristics which meet the requirements of customers and thereby provide product satisfaction. "Conformance to requirements"

Quality consists of freedom from deficiencies. "Fitness for use”[6].

A number of factors are used to assess the quality of software. These factors are divided into external and internal quality criteria. External quality is concerned with what a user experiences when running the software in its operational mode. Internal quality is referred to aspects that are based on development phases, and that are not visible to the end-user. External quality is significant to the user, while internal quality is important to the developer only. Usability is a basic quality property and one of the essential characteristics, which is necessary exists to ensure

DOI : $10.5121 /$ ijsea.2016.7202 
the success of any software product. It is a part that combines science and art and is not limited only on the web and websites, but that usability intervention in everything interacts with human. User Experience is software designs that focus on requirements, needs and user expectations to find effective solutions to the problems faced during use.

In this research paper, we provided an approach to insurethe improvement of the usability of software applications during the design phase.

\subsection{Software Usability}

The models of software quality are based on defining software quality in a term that is too abstract to be understood directly, by dividing it into attributes (usually called quality factors). One of these factors is usability. Each factor is composed of attributes (usually called characteristics) [1]. Usability is a measure of the quality of a person's experience in interacting with software product.

Usability is an essential factor of software quality. Usability has permanently been exists in the key models of software quality. Certainly, usability represents one of many factor affecting software quality [1].

\subsection{What is Usability?}

Software usability refers to the quality of a user's experience when interacting with software products or systems, including websites, software, devices, or applications. Usability is "about effectiveness, efficiency and the overall satisfaction of the user" [3].

It is important to understand that usability is not a single, one-dimensional property of a product, system, or user interface. 'Usability' is a combination of factors including [3]:

- Intuitive design: a nearly easy understanding of the architecture and navigation of the software or website

- Ease of learning: How fast a user who has never seen the user interface before can accomplish basic tasks?

- Efficiency of use: How fast an experienced user can achieve tasks in the system?

- Ability to remember (Memorability): How can the user remember to use the system effectively in future uses?

- Error occurrence and acuteness: to what extent users make errors while using the software, how serious the errors are, and to what extent users recover from the errors?

- User satisfaction: to what extentthe user likes using the system? 


\subsection{Importance of Usability}

Usability represents the measurement of the quality of a user's experience when interacting with a software product, whether a web site, software application, mobile technology, or any useroperated device.For the software, usability is a required condition for being. If the software or aweb site is difficult to use, people leave. If the software product fails to achieve a user requirements or the homepage fails to clearly state what a company offers and what users can do on the site, people leave.If users get lost on a web site, they leave. If a web site's information is hard to read or doesn't answer users' key questions, they leave. There's no such thing as a user reading a software or web site manual or otherwise spending much time trying to figure out an interface.There are plenty of other web sites available; leaving is the first line of defense when users encounter a difficulty [2].

\subsection{Who is the Beneficiary from Usability?}

Usability can be exploited by both users and developers. Users can benefit from usability since, they will be satisfied, not frustrated, with the web site or software product. As well as, they will enjoy interacting with the web site or software product. Users achieve their objectives effectively and efficiently. Furthermore support confidence and trust in the software product or web site. When the users are satisfied, they become dependable, and may even recommend your product or service to others [2].As a developer, may benefit from usability in many methods, such as, reduced development time, costs, support costs, user errors, training time and costs.

\section{USER EXPERIENCE}

User experience emphases on a deep understanding of users, what theirrequirements, what they value, their abilities, and also their limitations. It also takes into account the business objectives and objectives of the group managing the project. User experience practices encourage improving the quality of the user's interaction with and perceptions of software product and any related services.

The mainobjective of UX is to make sure that users catch value in what you are providing to them. In order to be a significant and valuable user experience, information must be[3]:

- Useful: the content should be original and fulfill a need

- Usable: the software product or website must be easy to use,Ease of use remains lively, and yet the interface-centered methods and perspectives of human-computer interaction do not address all dimensions of the design. In short, usability is necessary but not sufficient.

- Desirable: design elements such as image, identity, and brand are used to bring out emotion and appreciation

- Able to be found: Information needs to be navigable and locatable onsite and offsite

- Able to be accessed: Information needs to be accessible to people with disabilities 
International Journal of Software Engineering \& Applications (IJSEA), Vol.7, No.2, March 2016

- Reliable: Users must trust and believe what you tell them.

\subsection{What is User Experience Design?}

It's very important to understand the nature of UX Design. Unfortunately it gets easily confused with visual design and usability.A UX designer's is often lies at the crossroads of art and science and requires both extremely acute analytical thinking and creativity [4].

User Experience Design (UXD) is the process of realization user satisfaction by improving the usability, accessibility, and pleasure provided in the interaction between the user and the product.[7] User experience design enclosed traditional human-computer interaction (HCI) design, and extends it by considering all aspects of a product or service as perceived by users.[8]

\subsection{Users as the Centre of UX Design:}

In the case of we consider the core of UX design is the concept of constant iterative optimization, then the problem isthe product that the user is using. The problem of future users Spot it, define it, feel the pain it causes and eliminate it. That's the fastest way to great user experience. To stay on the same direction we'll need a lot of understanding and analytical skills, because the problematicissue with problems is that we sometimes have difficulty defining them - even if they trouble us.[4].

The key to success is to actually get to know the needs of your customers. Arm yourself to understand and talk to them. Get out of the building and face the problems that might be the foundation of your business. User experience design is deeply human centric [5].

\subsection{User Experience Design Best Demonstrated Practices to Improvingusability:}

In what waythe application developers can make great user experiences that are useful, useable, and desirable? To realize that, we have to reviewed applicable design to usability, there are four best practices:

- Users become know how the design appropriate for their needs.

- Firstly, the design should be done to avoid leaving user experience to chance.

- Trust no one — check to make sure users are satisfied[5].

\section{PROPOSED APPROACH FOR IMPROVING USABILITY}

This section presents an approach to improve the usability in software products. Usability of software applications enables the user to use the software with ease and flexibility to achieve its objectives, which designed the product for.

It is essential that the product be programmatic and practical usable. In addition it is an efficient and rapid tasks and performance efficiency. User interaction is easy and meets the needs and wishes of the declared and undeclared.

Usability property is one of the important characteristics of the software quality .It is considered as one of the characteristics that we judge by the success of software applications. This may represent the basic property in the continued survival of the software application alive.The perfect 
design of the user interface is the ideal and the most effective way to produce high usability give the user satisfaction and meet their needs and enable it to perform his work with ease and fun and effective..

Usability means the ability to easily use the software in order to achieve a particular goal. Usually it refers to the elegance and clarity in the design, whether normal software product or a website or an ordinary computer program to lead to efficient interaction and this term is an integral part of the User experience (UX). UX is defined as what is felt by the user when using the site or program. It is concerned in the techniques and tools that are used to create a good impression of the user.

As the usability and user experience are two sides of the same coin, we need to improve usability during the design phase. Hence, we proposed a framework for improved usability in software. Figure 1 shows the mechanism of the proposed framework:

- In the start the designer makes initial samples in the form of software interfaces, these interfaces submitted to the customer in the form of a set of data and functions that belong to these interfaces.

- Samples are included with the Preliminary list of questions and inquiries about the opinion used in these samples and apply this approach to all system interfaces.

- The designer creates interfaces initial system based on the requirements document, in addition to a list attached to a set of questions pertaining to this interface and are delivered to the customer by the designer.

Initially, the designer will explain to the customer the function of this interface. This is done either written through specific interface of system interfaces or through a direct dialogue with the user to clarify a working mechanism of the proposed document interface. What are the functions they offer and how they work? If there are functions lead to call other interfaces, we have to show and explain it to the customer, the designer taking into account the details of how to perform each function within the interfaces.

A list of questions is given to the customer concerning this interface. It is a comprehensive question about the interfaces as a unified system of any scale for all interfaces. Customer answers to these questions, then the designer to review the questions and find out the customer's opinion in the interface and learn about his proposals and his point of view in the primary interface. Design is revisedin accord with the customer requirements and recommendations. Then re-send after the new interface design to the customer again, accompanied by a list of questions after ending the work of the designer in a second sample from the same interface.

The goal of this interface is to identify the initial impression to the customer. What he wants and how he wants to see his system. What are the functions and data needs and data that cannot declare them directly?

By using this method we can get to what the customer wants with ease. And understand what he wants to see in its system with ease and convenience and transparency and not to complicate it. Re-design the interface until you get to the customer's satisfaction and we get to the interface that meets the needs and therefore we have achieved the customer's desire through repeated design of 
the interfaces according to their desires and needs. Figure 1 illustrates the steps of the framework for improving the usability of the software.

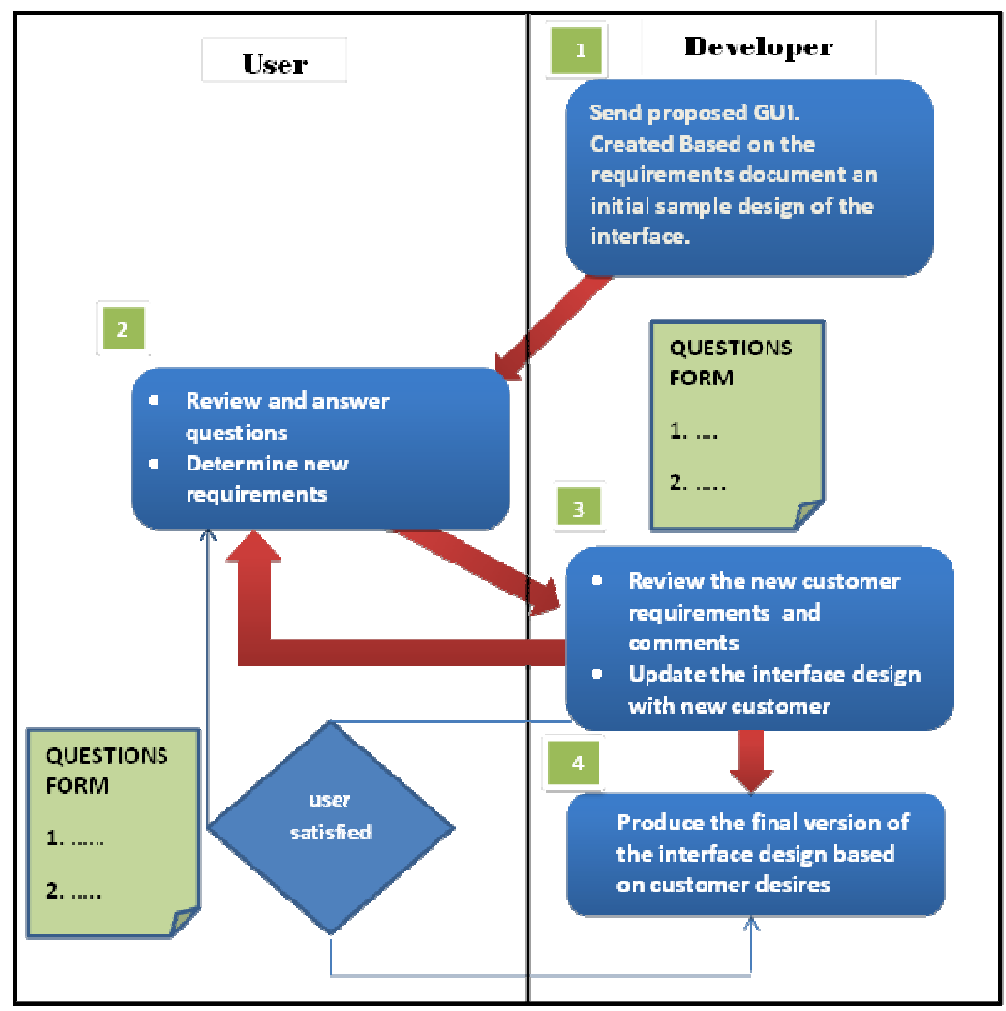

Figure 1: The proposed framework to improve the usability of the software.

\subsection{Case Study}

First, it is the establishment of the initial interface of the selected function. The developer designs the interface based on the document requirements attached a list of questions pertaining to this interface, also accompanied by an explanation of the functions of this interface and functions that they contain a full explanation with details to be understood by the customer. From here comes the role of the customer in improving the design of interfaces if there is a shortage or unwanted additions byansweringtheattachedquestions andexpress his opinion in them. The following interface entitled student data, and its functions:

Function "Add" means addition new student to the system. Added require to make sure that the new student does not already exist in the system records (to avoid repetition). This is done by the function "Search" where the student is entered in the "student database name." The system then searches for this student in the database. If the result is a message explains that "the student does not exist" then we can add all the required data according to their allocated fields. These fields are: student's name, passport number, nationality, date of birth, place of birth, country of residence, phone number and e-mail. If there is a field is not insert data the system displays a message indicating that states that "you should enter all fields" Then save the data by function "Save" and it will save all the student data in the student database. 
In the case the result was a message that states that "the student exists" where its data are displayed on the screen and we cannot add him because the student already exists in the system. Function "Delete" is another function carried out by the student data interface. We cannot delete the student's record from the system if the student does not already exist. Before holding this function enters a student's number or student's name and press the "Search" option. If the result is a message indicating that "the number exists" then we can delete the student data by pressing the function "Delete" and the system will delete all the student data from the database. If the result is a message states that "the number does not exist" then ensure that the student does not exist and we cannot delete its data.

The function "Update" is intended to edit student data exists in the system. We can through this interface change or update student data. To complete this function successfully, it must be a student registered in the database. It is searched first for the student by name or number, and then makes the appropriate change or update.

In this way the interfaces will be presented with a full explanation to the user so that he can perform his work with ease and proficiency. This work is to explain the interface mechanism and how the implementation of the tasks contained in this interface, in addition to the list of questions pertaining to this interface. These questions concerned with the characteristics of the interface during the design phase not in runtime. The questions as follows:

List of Questions concerning this system interfaces in general are as follows:

- Is the interface title expressed correctly?

- Is the data of the interface sufficient?

- Is there any data in the interface no need for its existence?

- Is the interface data are clear and correct?

- Is there a lack of data in the interface?

- Are all the functions of the proposed interface is sufficient and required?

- Are there important functions not mentioned in the interface?

- Are there any extra functions of the need and prefer to delete?

- Is the interface structure is good in terms of shape and color?

- Is satisfied with the user interface look ?

- Is the interface easy to understand? If the answer is "no" must be mentioned what is misunderstood data

- Is the interface easy to learn? If the answer is "no" must be mentioned why is difficult to learn.

- Is the interface attractive?

- Is the interface easy interaction from the user's perspective?

- Is there a relationship between the data addresses in the proposed interface? If the answer is yes identified these titles.

- Are there any interfaces or submenus you need of this interface?

- Is the interface need to display lists of data you want to work on it after saving or updating or deletion? Is the desired data displayed on the same interface or in another interface? 


\section{CONCLUSION}

By increasing the positive factors and follow the techniques and methods of effective and clear when managing software development helps to improve software product quality, customer service and achieve all their desires.

Paper prototyping is a useful technique for designers dramatically so that the design process smoothly and in a clear and explicit and simplified. On the part of design phase is benefit that the design has been based on the correct requirements that will be based on the user advertised or unexpected requirements. On the part of user it is gets a system that meets their needs and requirements in a logical and easy to understand, Ease of learning, Satisfaction, Efficiency, Memorability, Few errors and Ease of Use. In this paper we conclude, improving usability of the software is a user-centered design which is an iterative process. The work is carried on in a cycle of assumptions and evaluation, with a clear picture to users and design solutions to meet their requirements in completeness with each iteration. The proposed framework provides the practitioner with a set of characteristics in form of questions which can be used to organize and analyze information from users. They propose traceability from initial informationgathering during requirements setting and finally in evaluation. This mayallow the understanding of the specific needs about each feature to grow, or to be chance to verify whether the user requirements were chosen correctly or not.

\section{REFERENCES}

[1] MichalisXenos , M. Xenos, (2001), "Usability Perspective in Software Quality" Usability Engineering Workshop, Proceedings of the 8th Panhellenic Conference on In-formatics with international participation, Vol. 2, pp. 523-529, Cyprus.

[2] http://www.webnauts.net/usability.html, Last Access in 12/8/2015.

[3] http://www.usability.gov/, Last Access in 23/8/2015.

[4] MarcinTreder, (2013), "UX Design for Startups", Published in by UXPin On the web: www.uxpin.com .

[5] Mike Gualtieri, (2009),"Best Practices In User Experience (UX)", Gualtieri for Application Development \& Program Management Professionals, September 4, 2009.

[6] PatrikBerander, Lars-Ola Damm, Jeanette Eriksson, Tony Gorschek, Kennet Henningsson, Per Jonsson, Simon Kagström, DrazenMilicic, FransMartensson, Kari Ronkko, PiotrTomaszewski, (2005),Software quality attributes and trade-offs,Blekinge Institute of Technology.

[7] Kujala, Sari; Roto, Virpi; Väänänen-Vainio-Mattila, Kaisa; Karapanos, Evangelos; Sinneläa, Arto, (2011),"UX Curve: A method for evaluating long-term user experience". Interacting With Computers 23 (5): 473-483.doi:10.1016/j.intcom.2011.06.005. Retrieved 20 April 2014.

[8] "design in motion". IBM Design. Retrieved 2015-06-18. 